\title{
La normalización: base del análisis documental en los archivos
}

\author{
José Luis Bonal Zazo \\ Facultad de Biblioteconomía y Documentación \\ Universidad de Extremadura
}

\subsection{Resumen}

Se analiza todo el proceso que se ha llevado a cabo en la normalización de la desrcipción archivística, considerándose no solo los problemas que trae consigo la descripción propiamente dicha sino también los que aparecen por la existencia de normas de diversa índole. Posteriormente se realiza un análisis de la utilidad de este proceso y de las limitaciones, riesgos y exigencias que se producen en él.Al final se estudian los cambios que en el concepto mismo de descripción archivística se producen con motivo de la normalización.

Palabras clave: Descripción archivística. Normalización. Análisis documental. Normas de descripción.

\subsection{Abstract}

The evolution of archival description is analysed, giving special attention to the problems created by the coexistence of different standards regulating it. Thereafter, the achievements, limits, risks and exigencies of this process are considered. Finally, the changes that normalisation has provoked in the very concept of archival description is studied.

Keywords: Archival description. Normalisation. Document analysis. Description standards.

\section{Introducción.}

El proceso de normalización de la descripción archivística, iniciado en Estados Unidos a principios de los años 80, ha influido notablemente en el cambio del propio concepto de descripción. Mientras que tradicionalmente se equiparaba la descripción al proceso de elaboración de instrumentos de descripción (guías, inventarios y catálogos), hoy la descripción se considera una operación en sí misma, una operación de análisis documental, encaminada a la creación de 
representaciones exactas y concisas de cualquier unidad archivística, siempre bajo el respeto del principio de procedencia.

El interés por la normalización no es, sin embargo algo reciente. Ya Felipe II, en su Instrucción para el gobierno del Archivo de Simancas de 1588, al hablar de la realización del libro de los inventarios afirmaba que "las personas que adelante succedieren en el dicho cargo de archivero an de tener particular cuydado de hacer lo mismo cada uno en su tiempo".

En época más reciente, en la inauguración del primer Congreso Nacional de Archivos, celebrado en Sevilla en 1963 Antonio Matilla Tascón destacaba la conveniencia de normalizar en los archivos al decir que "los archiveros no pueden ser la excepción en la actividad de la vida presente, que exige, en todas las funciones, aparte de una mutua coordinación, la racionalización de los procesos y la normalización de las formas. Con ello se simplifican los métodos, se ahorra trabajo y tiempo en la ejecución y se obtienen mayores garantías en los resultados".

La preclara opinión de Matilla Tascón no es, pese a su anticipación, generalizable. Aunque han sido muchos los profesionales que se han manifestado a favor de las normas y, sobre todo, a favor de su aplicación a la descripción, el recelo aún persiste. A principios de la década de los noventa el prestigioso archivero francés Michel Duchein afirmaba: "se plantea a veces la cuestión: ¿sería posible hacer normas de carácter internacional para la inventariación de archivos? Mi contestación sería: no, en ningún caso. Normas de edición, sí. Quizás normas de indización. Pero bajo ningún concepto normas análogas a la existentes para la catalogación de libros".

El objetivo de este trabajo es analizar la problemática que entraña la normalización de la descripción archivística, así como examinar las repercusiones que las normas han tenido y tendrán en la descripción. Para ello se ha estructurado el estudio en seis partes. En primer lugar se analizan los problemas con los que tradicionalmente se ha encontrado la normalización en los archivos, para intentar, en segundo lugar, elaborar una propuesta de clasificación de las normas aplicables a la descripción archivística desde diferentes puntos de vista (su fuerza normativa, su origen y la profundidad de análisis de las normas). Los apartados tercero y cuarto están dedicados, respectivamente, al análisis de las ventajas que entraña la normalización y a la valoración de sus exigencias, límites e inconvenientes. En la quinta parte se describen, brevemente, cuáles han sido los hitos más importantes en el proceso de normalización descriptiva, para terminar, en sexto y último lugar, analizando las implicaciones que las normas tendrán en el propio concepto de descripción archivística. 


\section{Los problemas de la normalización en los archivos}

En el XII ${ }^{\circ}$ Congreso Internacional de Archivos, celebrado en Montreal en 1992, María Guercio planteaba la siguiente pregunta: "what are the concrete reasons why archivists have had difficulties in the past in creating and accepting standards? (Guercio, 1994, p. .201)". Las dificultades han sido numerosas, todas ellas caracterizadas por unas particularidades comunes, derivadas del carácter singular de los archivos y de la teoría archivística, frente a otros centros documentales.

La primera dificultad destacable es el propio objeto de los archivos y de la Archivística: el documento. Señala Pedro González que el carácter único del documento de archivo, frente al libro o la revista, ha determinado parcialmente el rechazo a las normas de descripción y ha llevado, en consecuencia, a la creación y utilización de normas específicas en cada archivo (González, 1991) (1). No es, sin embargo, la unicidad documental el único factor que ha determinado el alejamiento de las normas. Los documentos de archivo no son documentos independientes, sino que, al contrario, están fuertemente relacionados con sus productores, con las funciones por las que fueron creados y con su propia organización interna. Esta circunstancia, relacionada directamente con la base de la teoría archivística, influye notablemente en todas las operaciones relacionadas con su tratamiento. Por otra parte, las características descritas se ven complicadas por dos factores, en primer lugar, por el creciente volumen documental y la diversificación de sus formatos de presentación (Cox, 1994, p.170) y, en segundo lugar, por la heterogeneidad de los documentos de archivo, como consecuencia del elevado número de productores y de su diversidad a lo largo del tiempo. (Guercio, 1994, p. 201).

Junto a los documentos, también los usuarios han influido negativamente en el desarrollo de prácticas normalizadas (2). El prototipo convencional de usuario de archivo ha sido, durante mucho tiempo, el del investigador caracterizado por su erudición y amplio conocimiento de la materia estudiada, caracterizado también por su laboriosidad y paciencia en el trabajo y, sobre todo, como señala Xavier Tarraubella, porque: "no es refia de la informació o l'exhaustivitat dels inventaris realizats pels arxivers i vol accedir directament a la documentació arxivada, a las sèries documentals, sense la intermediació dels instruments de descripció" (Tarraubella, 1998. Se trataba, por tanto, de usuarios que apenas necesitaban de los archivos poco más que orden y conservación. A partir de los años 50 comienza a producirse un cambio trascendental en la tipología de usuarios, debido, fundamentalmente, al aumento del número de personas que acuden a consultar documentos, aumento que provocó una diversificación de los perfiles de usuarios (3). Esta diversidad repercutirá directamente en la obligación de uni-

Scire. 6:1 (en.-jun. 2000) 55-75. 
ficar prácticas y mejorar procedimientos de descripción, capaces de satisfacer todas las necesidades informativas de los usuarios.

Otro de los factores que han incidido negativamente en la difusión y utilización de prácticas normativas ha sido la tradicional forma de trabajo del archivero, generalmente aislada, independiente y con unos criterios propios, adaptados a sus necesidades específicas (Cox, 1994, p.173). Causas diversas, como el aumento del número de profesionales, la diversificación de su perfil, la proyección social de los archivos y, sobre todo, la influencia de disciplinas afines, han contribuido a la introducción de prácticas cooperativas en el ámbito de los archivos, prácticas que exigen el desarrollo de actividades normalizadas.

Otra causa que ha repercutido en la falta de normalización ha sido la diversidad existente en el ámbito de la Archivística, diversidad que se advierte tanto en cuanto a estructuras de organización y funcionamiento de archivos (Muñoz Feliu, 1995, p. 26), como en la existencia de diferentes realidades históricas, administrativas, culturales y de formación (Guercio, 1994, p. 201).

Junto a los factores enunciados, todos ellos de carácter teórico o profesional, cabe destacar también la existencia de razones económicas. La creación de normas técnicas exige contar con un fuerte respaldo económico. Frente a este requisito, la profesión archivística se ha caracterizado, tradicionalmente, por la falta de recursos (Cox, 1994, p.173).

\section{Diferentes criterios, diferentes tipos de normas}

Intentar hacer una aproximación general a la tipología de las normas existentes es difícil por varias causas.

En primer lugar, por la existencia de un concepto vago y difuso de qué es una norma, no sólo en el ámbito de los archivos, sino en otras muchas disciplinas (4). La ausencia de un concepto preciso dificulta su sistematización.

En segundo lugar, un elemento que obstaculiza la elaboración de una hipotética clasificación general de las normas, es la diversidad de criterios que pueden ser usados como base para establecer la tipología. García Gutiérrez, siguiendo parcialmente a González de Guzmán y a Verman, señala la existencia de cinco criterios básicos, en función de los cuales se puede abordar el estudio de la tipología de las normas: el carácter jurídico (público o privado) de la norma, su contenido, el ámbito de aplicación, los destinatarios de la norma y el grado de profundidad en la normalización (González de Guzmán, 1952 ; García Gutierrez, 1985, p. 59)

Por otra parte, el propio carácter cambiante de las normas, su evolución y su desarrollo impiden hacer una clasificación general, duradera en el tiempo.

Scire. $6: 1$ (en.-jun. 2000) 55-75. 


\subsection{Tipos de normas relacionadas con la descripción archivística}

En el ámbito concreto de la descripción archivística, existen varias propuestas de clasificación. Apunta Pedro González, siguiendo en buena medida a los autores norteamericanos, que la normalización de la descripción de archivos puede ser analizada desde diferentes puntos de vista:

- Documentos que permiten normalizar la estructura de los datos (responden a la pregunta: ¿cuáles son los elementos de la descripción?).

- Documentos para la normalización del contenido de cada uno de los elementos de la descripción (dan respuesta a las siguientes preguntas: ¿qué información incluir en cada elemento? y ¿de qué forma?).

- Documentos para la normalización de los instrumentos de descripción.

- Documentos normativos en el área de los lenguajes de indización (González, 1991, p.162) .

El Grupo de Trabajo sobre Normas de Descripción de la Society of American Archivists (SAA) estableció la existencia de varios tipos de normas, en función de tres criterios diferentes (Bearman, 1989) : el poder normativo del texto, su origen y la profundidad en la normalización de la descripción (fig. 1.).

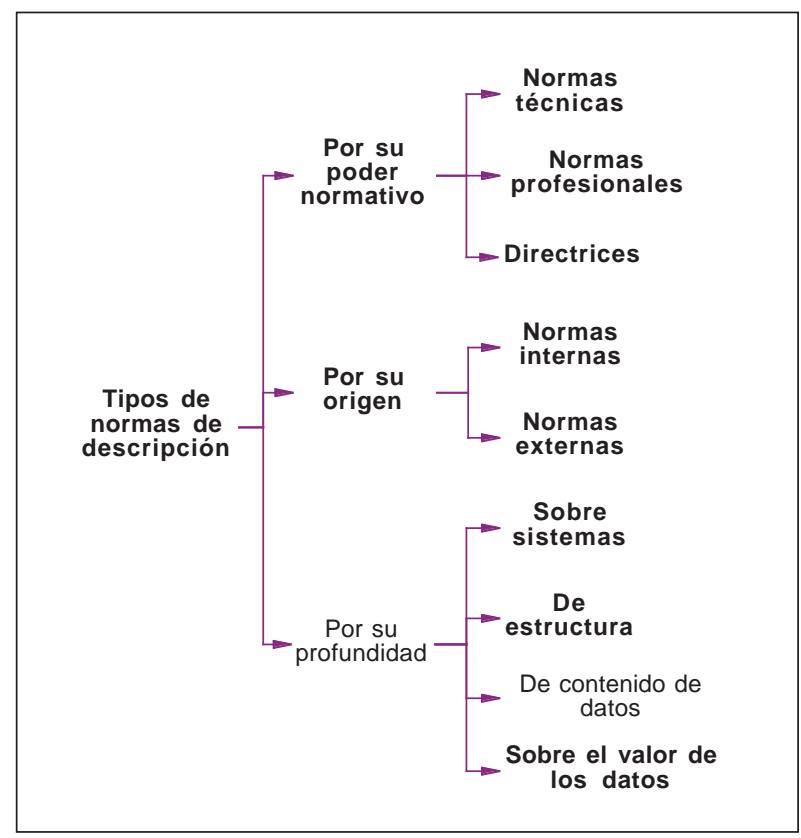

Fig. 1 Tipología general de las normas de descripción

Scire. $6: 1$ (en.-jun. 2000) 55-75. 


\subsubsection{Tipos de normas de descripción en función de su valor normativo}

Por lo que respecta al primer aspecto, el poder preceptivo de las normas de descripción, es posible establecer la existencia de tres tipos de documentos normativos:

1. Normas técnicas. Se trata de normas que comúnmente regulan el uso y la compatibilidad de equipamientos y que, en el caso de las Ciencias de la Información, se usan para regular el intercambio de información (Cox, 1994, p. 166) . Son las normas más rígidas, que, según Crawford: "is not subject to unilateral change without notice and which, if properly followed, will yield consistent results" (Crawford, 1986) (7). Aunque estas normas regulan numerosos elementos del trabajo cotidiano, incluido el trabajo archivístico (códigos de caracteres, sistemas informáticos, etc.), todavía no se ha desarrollado una norma de este tipo específica para el trabajo archivístico, aunque sí existen adaptaciones de normas técnicas generales procedentes de otros ámbitos (Working Group..., 1989, p. 456-461) .

2. Normas profesionales (convenciones o reglas). Las normas profesionales son normas que regulan prácticas y procedimientos de trabajo. Se trata de normas más flexibles que las anteriores y que se acomodan mejor a las prácticas locales. No obstante, pueden tener el mismo valor normativo que las normas técnicas si se aplican estrictamente (Ibidem, p. 167) . En este caso, a diferencia de las anteriores, sí que existen normas creadas específicamente para la descripción archivística, tales como la ISAD $(\mathrm{G})$, RDDA, MAD2, APPM e ISAAR(CPF). Tradicionalmente han sido consideradas por los archiveros como las normas más importantes y también las más deseadas.

3. Directrices. Se trata de instrucciones generales o recomendaciones, que no tienen el mismo valor normativo que las anteriores. Nancy Bolt establece que la diferencia de las directrices con los documentos normativos anteriores es que: "there are consequences of not meeting" (Bolt, 1988)(8) . Tal como afirma Cox las directrices son un paso previo para el desarrollo de normas más específicas (profesionales o técnicas). No obstante, frecuentemente, es difícil establecer la frontera entre unas y otras.

\subsubsection{Tipos de normas de descripción en función de su origen}

En cuanto a su origen, se puede hablar de dos tipos de normas: normas externas, es decir, normas procedentes de ámbitos de trabajo diferentes del propiamente archivístico; y normas internas, normas creadas específicamente para el 
desarrollo de trabajos de archivos (Working Group..., 1989, p.456-461) . Normas como el formato USMARC AMC, RDDA, MAD2, ISAD $(G)$ o APPM, son normas de carácter interno, mientras que otras, como las AACR2, son normas externas (9). No obstante, no se puede establecer una división tajante entre normas externas y normas internas, algunas, como APPM, aunque son de carácter interno, tienen una fuerte influencia de disciplinas externas a la Archivística.

\subsubsection{Tipos de normas en función del nivel de descripción}

Dependiendo de la profundidad alcanzada en la normalización existen, según David Bearman, cuatro tipos de normas (Bearman, 1989) (10):

- Normas sobre sistemas de información. Son las más generales, ya que definen las funciones de cada componente dentro de un sistema de información global, tanto si es un solo archivo como si es una red o un sistema de archivos (Lemoine, 1994).

- Normas de estructura de datos. Se trata de normas que definen los elementos informativos que deben ser recogidos en los componentes de un sistema de información; este tipo de normas sólo regulará los datos que es necesario incluir en un sistema de información, pero no su contenido, son normas relativas al formato externo de los datos. Un claro ejemplo de este tipo de normas es el formato USMARC AMC (11).

- Normas de contenido de datos. Son las normas que regulan qué información introducir en cada elemento definido en las normas de estructura de datos, son normas tales como las AACR2, APPM, MAD2, ISAD $(G) 0$ ISAAR(CPF) (Weber, 1989, p. 509-510) .

- Normas sobre el valor de los datos. Proporcionan listas o tablas de términos, nombres, códigos alfanuméricos u otros datos usados en elementos particulares de estructuras de datos; se trata de todas aquellas listas de materias, tesauros, listas de autoridades, listas de nombres propios, etc., que recogen la forma, ya establecida, de los términos que pueden ser usados para la descripción, sin dar lugar a la interpretación.

Como señala María Guercio, las dificultades para normalizar los datos con la finalidad de crear sistemas de información archivística, se encuentran no sólo en el nivel de contenido de datos, sino también en el de estructura de datos (Guercio, 1994). Un programa general de descripción debe abarcar, por tanto, las distintas categorías apuntadas.

\section{Las ventajas de la normalización}

La justificación de la utilidad de las normas se encuentra en su propia naturaleza; tal como señala García Gutiérrez, una norma siempre debe: "repercutir 
positivamente en el bienestar y progreso de la sociedad" (García Gutierrez, 1989, p. 58), las normas son, por esencia, beneficiosas.

Por lo que respecta a la normalización de la descripción, las normas presentan importantes ventajas. En primer lugar, ventajas para los usuarios, debido a que favorecen la comprensión de las descripciones. Ventajas, en segundo lugar, para la teoría archivística, ya que contribuyen a la comunicación y la cooperación entre los archivos, tanto a escala nacional como internacional. En tercer lugar, la aplicación estricta de normas es ventajosa para el acceso a los documentos; y, como consecuencia de todo lo anterior, las normas conllevan ventajas económicas derivadas de la mejora de la calidad de los servicios.

La principal utilidad de las normas, desde el punto de vista de los usuarios, es la de favorecer la comprensión de las descripciones. Si partimos de la idea de que la descripción es una representación de una unidad documental, convendremos en que esta representación, debe proporcionar información en cantidad suficiente y forma adecuada para que los usuarios decidan si la documentación es relevante para sus necesidades (Szary, 1989, p. 520), siempre respetando tres objetivos básicos: 1) preservar la integridad de los archivos; 2) comunicar la información que contienen y 3 ) facilitar el acceso a todos aquéllos que tengan derecho a él (Haworth, 1992, p.188). Como señala Haworth, las normas constituyen una especie de "gramática especializada" que regula, de forma precisa, la disposición, contenido y uso de los elementos informativos, evitando así la creación de descripciones e instrumentos de descripción incoherentes, inconsistentes, incomprensibles y, como consecuencia, confusos (Haworth, 1992, p. 189) . De este modo, se facilita a los usuarios la consulta de instrumentos con unas características y contenido homogéneos y de mayor calidad (Steinmark, 1996, p. 63).

Además de los usuarios, son los propios archivos y archiveros los beneficiarios indirectos de la normalización. La aplicación de las nuevas tecnologías al almacenamiento e intercambio de información facilita la comunicación y la cooperación archivística, pero exige, como contrapartida, la utilización de normas uniformes. Como señala Szary, es necesario que: "the profesion has embarked on a shared enterprise that requires unambiguos communication"(Szary, 1989, p. 520). Las repercusiones de la normalización son notables para el trabajo archivístico, ya que favorece el desarrollo de iniciativas de cooperación no sólo con otros centros de archivos, sino también con otros profesionales de la información, con sus técnicas y sus equipamientos (11). La utilización de las grandes redes bibliográficas norteamericanas para la difusión de descripciones archivísticas constituye un buen ejemplo de esta utilidad. Se puede afirmar que la normalización contribuye, de este modo, a la universalización de los problemas y de las prácticas archivísticas (Cox, 1994, p.169), favoreciendo, también, la búsqueda de soluciones globales.

Scire. $6: 1$ (en.-jun. 2000) 55-75. 
En cualquier caso, independientemente del uso de las normas en contextos automatizados, compartidos o no, o incluso en sistemas manuales, la normalización puede actuar como instrumento de seguridad para garantizar una alta y homogénea calidad en la descripción, con los beneficios que ello puede reportar en el trabajo interno de gestión de fondos (Steinmark, 1996, p. 63).

En opinión de Richard Szary, las normas presentan otra ventaja, directamente relacionada con las ya apuntadas: la mejora en el acceso al contenido de los documentos, a través de la normalización de los elementos que han de servir como puntos de acceso (Szary, 1989, p. 520).

En definitiva, las ventajas enunciadas influyen directamente en la calidad de los servicios (12), con el provecho económico que ello conlleva. Como destaca el Grupo de Trabajo sobre Normas de Descripción de la SAA: "economic benefits are the primary incentive behind the development and successful implementation of standards... The project cost/benefict ratio will have a greater impact on a decision to pursue a standard or not than any other factor"(Working Group..., 1989, p. 452).

\section{Limitaciones, exigencias y riesgos}

Pese a las ventajas, el uso de las normas entraña también limitaciones, exigencias y riesgos.

La primera exigencia es la necesidad de consenso: las normas, para ser efectivas, no pueden ser dictadas y establecidas por una fuente centralizada, sino que deben ser resultado de la consulta a los profesionales afectados por su uso y fruto del consenso entre los mismos (Szary, 1989, p. 522). La elaboración y mantenimiento de normas exige, por tanto, la existencia de una infraestructura mínima que se encargue no sólo de su redacción, sino también de su difusión, revisión y actualización. Como señala Lisa Weber, las normas no son estáticas (Weber, 1989, p. 512): una norma puede presentar ambigüedades y omisiones, o, simplemente, pueden cambiar las circunstancias de su utilización o exigir adaptaciones a las prácticas locales (Szary, 1989, p.522).

Otro de los factores que ha contribuido a la diversificación de campos de actuación, y a la complicación del panorama normativo, es la gran cantidad de instituciones con potenciales competencias en este campo. El Grupo de Trabajo sobre Normas de Descripción de la SAA cita más de treinta entidades con atribuciones normativas (asociaciones, entidades de la administración, organismos de normalización, etc.). Todas ellas pueden ser clasificadas en tres tipos: 1) organizaciones e instituciones formadas básicamente por archiveros; 2) organizaciones e instituciones relacionadas con el ámbito de los archivos y, 3) organizaciones nacionales e internacionales de normalización (Working..., 1989, p. 456-461) 
(13) . En cualquier caso, tal como afirma Haworth, corresponde a los archiveros dirigir el proceso de normalización en su disciplina (Haworth, 1994, p. 193).

Un factor que complica aún más esta situación es la existencia de normas que afectan, en conjunto, tanto a las comunidades de archiveros como de bibliotecarios (Weber, 1989, p. 512).

En el caso particular de los archivos, la falta de tradición normativa en la profesión entraña el riesgo complementario de caer en la simplificación del contenido de los elementos descriptivos. El desarrollo de actividades de normalización exige, por tanto, establecer de antemano la forma precisa del contenido de cada elemento y su utilización (Szary, 1989, p. 520). Lisa Weber va más lejos en esta idea, al afirmar que antes de normalizar la descripción conviene analizar y establecer cuáles son los objetivos de la misma: "it is difficult if not impossible to develop adequate standards to guide the development of archival description when we are so vague about its very purpose" (Weber, 1989, p. 511). En la práctica, la mayoría de las obras normativas difundidas aparecen precedidas de estudios teóricos, más o menos amplios, sobre sus principios y su contenido (Ibídem, p. 512) .

Apunta Lisa Weber un problema más, específico de la normalización archivística: ¿cómo conseguir hacer cumplir las normas? Así como las normas industriales son de uso casi obligatorio por razones económicas, no hay ninguna razón que obligue al uso de las normas de descripción. Como señala García Gutiérrez, diversos factores subjetivos, de carácter político-ideológico, pueden incidir negativamente en los procesos de normalización que afectan a la información científica (García Gutierrez, 1985, p. 66). Únicamente la participación en proyectos cooperativos puede contribuir a la obligación de usar las normas. Es el caso, por ejemplo, de RLG y de OCLC, instituciones que establecen la obligación de usar el formato USMARC AMC, las Reglas de Catalogación Angloamericanas o APPM y la Lista de Encabezamientos de Materia de la Biblioteca del Congreso.

Las normas descansan sobre la base de la experiencia, por esta razón, su difusión y su aceptación dependen, en buena medida, de que éstas recojan la práctica que era común antes de su aparición, como señala Gagnon-Arguin: "les normes, qui peuvent paraître contraignantes et imposées de l'exterieur, sont en fait la généralisation et la systématisation d'une pratique" (14).

Pero además de su origen, otros factores influyen en su difusión, Según Richard J. Cox, entre éstos cabe destacar los siguientes:

1. El desarrollo de actividades de educación y formación que incluyan la normalización entre sus materias de enseñanza. 
2. La investigación sobre la naturaleza, los principios y los elementos de las normas de descripción.

3. La promoción de nuevas iniciativas de normalización por parte de organismos que influyan en su aplicación.

4. La creación de comisiones técnicas de carácter internacional que favorezcan la difusión de las normas ya existentes y trabajen en la redacción de nuevas normas.

5. El fomento de las estructuras que intervienen en la promulgación de instrumentos normativos.

Uno de los factores que más ha influido en la expansión y la aplicación de las normas ha sido el desarrollo tecnológico. Son numerosos los autores que consideran que la normalización es resultado, o efecto, de la incorporación de las nuevas tecnologías a las actividades archivísticas. Stefano Vitali, por ejemplo, afirma que: "L'avvio del dibattito internazionale sulla normalizzazione della descrizione è probabilmente uno degli effetti più significativi delle ripercussioni che l'avvento delle tecnologie informatiche ha provocato sul mestiere dell'archivista" (Vitali, 1994, p. 303) (15).

Está generalmente aceptado que la irrupción de las nuevas tecnologías en el mundo de la información ha cambiado los modos de trabajo (Fishbein, 1981), según Charles M. Dollar, la presencia de las nuevas tecnologías afecta a tres aspectos: la sustitución, como soporte de información, del papel por soportes electrónicos (16), el desarrollo de las redes de comunicaciones y, como consecuencia de lo anterior, el desarrollo de normas específicas que faciliten la interconexión de diferentes sistemas informáticos para facilitar el intercambio de información (Dollar, 1993, p. 39). Son éstos los dos aspectos que más afectan a la descripción archivística. Todo ello está llevando, de algún modo, a borrar los límites de las disciplinas y profesiones y unificar funciones (Dollar, 1994, p. 449450).

\section{La normalización de la descripción}

Afirma María Guercio que: "rules of description have always existed in archival work". Efectivamente, reglas mínimas de descripción siempre han existido. Eran, sin embargo, pautas aisladas, que respondían a necesidades locales y se ocupaban de aspectos tan diversos como los principios de la descripción, los elementos que debían recoger los instrumentos de descripción, la forma de disposición de los datos..., pero siempre de forma particular (Guercio, 1994, p. 202203). Con la finalidad de presentar el proceso de normalización de la descripción, apuntaremos brevemente cuáles han sido los principales hitos en la aparición de 
normas profesionales, técnicas y de contenido, normas en cuyo estudio profundizaremos en el resto del trabajo.

\subsection{Normas profesionales (17)}

Aunque existen numerosos precedentes, consideramos que la actividad de normalización descriptiva específicamente archivística, que desembocó en la creación de las primeras normas de descripción internacionales, se inició en Estados Unidos a principios de los años ochenta, cuando, en 1982, Hensen adaptó varios capítulos de la $2^{a}$ edición de las Reglas de Catalogación Angloamericanas (en adelante AACR2) para la descripción de documentos de archivo, si bien es verdad que ya las propias AACR2 disponían de un capítulo con tal fin (Reglas..., 1983). La obra de Hensen, Archives, Personal Papers and Manuscripts (APPM), fue respaldada por la Society of American Archivist y por la Library of Congress, adquiriendo así naturaleza normativa (Hensen, 1989).

Pocos años después, en 1986, Michael Cook publicaba en Gran Bretaña un manual de descripción de similares características al de Hensen, el Manual of Archival Description (MAD); el manual británico, con un contenido mucho más detallado que APPM y una disposición distinta de los datos, adquiriría su contenido y estructura definitiva en la segunda edición (MAD2) (Cook, 1989), y seguiría un proceso paralelo a la obra de Hensen: nació como un manual de descripción resultante de una iniciativa particular, para convertirse, posteriormente, en fuente normativa. No obstante, la obra de Cook tuvo mucha menos difusión práctica que la de Hensen, aunque su influencia teórica fue notablemente mayor.

A finales de los 80, los archiveros canadienses publicaron la tercera gran obra normativa de carácter nacional: las Règles pour la Description des Documents d'Archives (Regles..., 1990) . Como el manual de Cook, las RDDA tenían un articulado extenso y detallado. Al igual que el manual de Hensen sufrían una fuerte influencia bibliográfica, fruto de su origen común en las AACR2. Sin embargo, a diferencia de ambos, era la primera obra que nacía con todas las características formales de cualquier norma: era resultado del consenso profesional, creada por un organismo nacional, con un fuerte carácter técnico y de libre aplicación.

El desarrollo normativo norteamericano y británico, fue decisivo, y en buena medida influyente, para que el CIA, a principios de los 90 emprendiera el trabajo necesario para la elaboración de una norma internacional de descripción archivística, norma que aparecería en 1993, bajo el título de International Standard Archival Description (General) —ISAD (G) - (Consejo..., 1995), a la que seguiría, en 1995, una norma específica para la normalización de los puntos de puntos de acceso, la International Standard Archival for Authority Record (Corporate bodies, Persons and Families), la ISAAR(CPF). En 1999, el Comité

Scire. $6: 1$ (en.-jun. 2000) 55-75. 
sobre Normas de Descripción, reunido en Estocolmo, aprobaba la segunda edición de la Norma $\operatorname{ISAD}(\mathrm{G})$, tras cinco años de prueba y de recogida de propuestas y sugerencias (Consejo..., 2000) (18).

En España existen diversas iniciativas para la aplicación e implantación de las Normas internacionales. De todas ellas destaca el proyecto MDM (Manual de Descripción Multinivel) (Manual, 2000), un proyecto emprendido por los archiveros de Castilla y León, con el objetivo de crear un cuerpo normativo general, aplicable a la descripción archivística, que integre los principios internacionales de la descripción con las prácticas de trabajo españolas. En una primera fase está prevista su difusión entre los profesionales con la finalidad de probar la viabilidad de su aplicación.

\subsection{Normas técnicas}

Junto a la definición y disposición de los elementos que integran la información archivística, otro campo de actividad normativa ha sido la creación de formatos específicos para el intercambio de descripciones archivísticas en soporte informático. El formato creado, perteneciente a la familia de los MARC, el USMARC AMC (United States MARC for Archival and Manuscripts Control) fue aprobado en 1983 (Sahli, 1985). Su aplicación se ha limitado sólo a Estados Unidos. Fuera de este país, el MARC sólo se ha utilizado, de forma masiva, en Canadá, donde se usa el mismo formato que se emplea comúnmente para el intercambio de información bibliográfica, el CAN/MARC. Junto a los anteriores, algunos países europeos han puesto en marcha proyectos experimentales, proyectos que, en cualquier caso, carecen de la proyección existente en Norteamerica. Uno de los intentos destacables ha sido el de Gran Bretaña, donde se intentó crear un formato MARC específico para archivos, compatible con el MAD2.

Una línea de normalización, mucho más reciente, es la codificación de los instrumentos de descripción en formato digital. El proyecto inicial, el Encoded Archival Description, iniciado por la Universidad de Berkeley en 1993, está concebido para reproducir en formato digital la estructura jerárquica de los instrumentos de descripción (García Martínez et al., 1998).

\subsection{Normas de contenido}

Son numerosos los tesauros y listas de materias que se han creado en el ámbito de los archivos, y su producción continúa aumentando. Sin embargo, frente a la repercusión internacional de las normas profesionales y las normas técnicas, éstos han tenido una difusión mucho menor, debido a que, en buena medida, responden a prácticas locales y, ocasionalmente, son instrumentos compartidos con las bibliotecas. 


\section{7. ¿Hacia un nuevo concepto de descripción?}

Aunque la operación de describir es única, las definiciones del término son tan numerosas como manuales, estudios, léxicos y diccionarios se han ocupado de su significado. Las propias obras normativas proponen nuevas definiciones que, por su forma y su contenido, aportan un nuevo enfoque al concepto. Su análisis, exige un breve recorrido por las definiciones precedentes, con el fin de determinar si la normalización ha supuesto un cambio del concepto de descripción archivística.

\subsection{El concepto de descripción antes de la normalización}

La mayoría de las obras que se han ocupado de la descripción archivística con anterioridad a la aparición de las normas internacionales, han definido la descripción a partir de los tres elementos básicos siguientes:

1. la actividad desarrollada (la reseña de los caracteres internos y externos de los documentos);

2. el resultado de la actividad (los instrumentos de descripción);

3. el objetivo de la descripción (facilitar el acceso al contenido de los documentos) (19).

Buena parte de las definiciones existentes antes de la difusión de la Norma $\operatorname{ISAD}(\mathrm{G})$, y de las obras normativas nacionales, se basan, para definir la descripción documental, en alguno, o algunos, de los tres elementos indicados. Frecuentemente en los dos últimos. Dependiendo de la importancia dada a uno u otro, se pueden diferenciar dos corrientes: la primera equipara la operación de describir a la realización de instrumentos de descripción (20), mientras que, según la segunda, la descripción es la actividad destinada a la representación de los documentos (Champagne, Chouinard, 1987, p. 93).

\subsection{El concepto de descripción propuesto por la norma ISAD(G)}

A diferencia de la mayoría de las obras precedentes, la norma $\operatorname{ISAD}(\mathrm{G})$ propone una definición de descripción diferente, que parte de unos presupuestos novedosos.

Ya en 1986, los miembros del Bureau Canadien des Archivistes, influidos por el proceso de normalización descriptiva iniciado en su país, definieron la descripción archivística como la representación más exacta y más concisa posible de cualquier unidad archivística, elaborada siempre bajo el respeto del principio de procedencia. Fuertemente influidos por este concepto de descripción, los responsables de la elaboracion de la norma $\operatorname{ISAD}(\mathrm{G})$ establecieron que la descripción es: "la creación de una representación exacta de una unidad de descripción y de las partes que la componen, en caso de haberlas, por el procedimiento de capta- 
ción, cotejo, análisis y organización de cualquier información que sirva para identificar la documentación de archivo y explicar el contexto que la produjo" (Consejo..., 1995). La nueva definición rompe con algunos de los planteamientos expuestos en las definiciones precedentes, pero, en contraposición, incluye elementos plenamente aceptados en la práctica y no incluidos en la teoría.

De forma general, las aportaciones de la definición propuesta por ISAD (G) se pueden resumir en los siguientes puntos:

- Disociación entre los conceptos de "descripción" e "instrumento de descripción”. La definición que propone la $\operatorname{ISAD}(\mathrm{G})$ separa completamente, y a diferencia de las definiciones precedentes, la descripción (actividad) de los instrumentos de descripción (resultado). Los instrumentos de descripción pueden ser uno de los diferentes resultados posibles de las descripciones obtenidas, pero el objeto de la descripción no es ya la elaboración de instrumentos de descripción, como planteaban las definiciones anteriores, sino la elaboración de representaciones precisas de unidades de descripción; representaciones que, en un contexto automatizado, pueden tener diferentes formatos de salida y dar lugar, por tanto, a diferentes instrumentos de descripción, aunque el objetivo de la descripción no es ya su elaboración (21).

- Inclusión del principio de procedencia en la descripción. Tanto las normas nacionales precedentes como la propia $\operatorname{ISAD}(\mathrm{G})$ unen, indisolublemente, la descripción al principio de procedencia. La obligación de incluir información relativa al contexto de creación de los documentos en la descripción supone la necesidad de proporcionar datos sobre el productor de los mismos. No obstante, ya antes de la publicación de la $\operatorname{ISAD}(\mathrm{G})$ era práctica común incluir información relativa al contexto de creación en algunos instrumentos de descripción, así como elaborar instrumentos de descripción que respetaran la procedencia y siguieran el cuadro de clasificación; la verdadera aportación de las normas, en este sentido, ha sido elevar a nivel normativo esta práctica, estableciendo la obligación de su cumplimiento (22).

- Aumento del número de elementos informativos. Todas las normas amplían, potencialmente, la información que debe recoger una descripción archivística: cualquier información que permita identificar la documentación y explicar el contexto de creación puede ser considerada información descriptiva y como tal puede ser incluida en la descripción.

La influencia de la definición propuesta por $\operatorname{ISAD}(\mathrm{G})$ será inmediata, algunas obras aparecidas después de la publicación de la norma van a adoptar inmediatamente el nuevo concepto de descripción. Así, el nuevo manual de archivís- 
tica francés, bajo el epígrafe "un nouveau concept", ya recoge la idea de que: "la description archivistique a pour object de donner de toute unité archivistique a décrire la représentation la plus exacte et la plus concise possible, dans le respect du principe de provenance" (Favier, 1993, p. 141).

En conclusión, como respuesta a la cuestión que planteábamos al comienzo de este epígrafe-ila normalización está contribuyendo al cambio del concepto de descripción archivística? —, se puede afirmar que las normas, junto con las nuevas tecnologías, han contribuido a aclarar y a precisar conceptos, terminología y pautas de actuación relacionadas con el trabajo de la descripción archivística. Contribuyendo también a la introducción de modelos procedentes de disciplinas relacionadas sin que se haya producido la pérdida de las características propias del trabajo archivístico.

\section{Conclusión}

La normalización está llevando, en la actualidad, hacia un nuevo concepto de descripción archivística: Tradicionalmente descripción era sinónimo de elaboración de instrumentos de descripción. En la actualidad, sin embargo, la descripción está tomando fuerza como una actividad en sí misma. El nuevo concepto de descripción documental es un concepto integral, fruto, en buena medida, de su relación con el principio de respeto a la procedencia y al orden original. De acuerdo a esta circunstancia, la descripción debe recoger todos aquellos datos, relativos al origen y tratamiento de los documentos, que puedan afectar no sólo a la comprensión del contenido de los documentos, sino también que puedan ampliar su información. Por otra parte, este nuevo concepto de descripción documental está sentando las bases para el desarrollo de la teoría sobre el análisis documental en lo archivos. Es previsible que en los próximos años se profundice en la investigación de un modelo propio de análisis documental, similar a los existentes en áreas de conocimiento afines, pero paralelo a aquéllos.

\section{Notas}

(1) También Louise Gagnon-Arguin y Richard J. Cox comparten esta idea. (Gagnon, 1992, p. 37 ; Cox, 1994, p.173)

(2) Con esta idea, apuntada por María Guercio, coincide Miguel C. Feliú, quien opina que el carácter restringido y minoritario de los usuarios puede explicar, entre otras causas, el retraso en la elaboración de una norma de descripción archivística (Muñoz, 1995, p. 26).

(3) Una clasificación de estos tipos de usuarios puede encontrarse en la citada obra de Tarraubella. Según este autor, llegarán a cuatro categorías: investigadores profesionales, investigadores aficionados, estudiantes y ciudadanos en general (Tarraubella, 1998, p. 192-195).

Scire. $6: 1$ (en.-jun. 2000) 55-75. 
(4) En el caso de la descripción archivística las tres "normas" nacionales generales existentes antes de la aparición de la $\operatorname{ISAD}(G)$ aparecen con el título de "Manual" o de "Reglas".

(5) Clasificación realizada a partir del estudio de David Bearman Description Standards: a framework for action. (Bearman, 1985)

(6) Cit. por García Gutierrez (1985).

(7) Cit. por Working Group... (1989, p. 456-461).

(8) Cit. por Working Group... (1989, p. 456-461).

(9) El formato USMARC AMC podría ser considerado como una norma de carácter externo, o al menos, intermedio, ya que parte del tronco común de todos los formatos MARC (Checklist..., 1989).

(10) Cit. por Working... (1989, p. 455-461)

(11) Para Kent M. Haworth el desarrollo del concepto de sociedad de información ha favorecido la relación de los archivos con otras instituciones documentales. El mismo autor advierte que es peligroso para los archiveros mantener una actitud agresiva, en la defensa de las diferencias de la disciplina, debido al riesgo de quedar desvinculado de la sociedad de la información (Haworth, 1994, p. 189-191).

(12) Tal como afirma Leopold Auer: "In a very general sense there may be identified two main reasons for standardization: the one is to ensure a certain level of quality; the other is to enable interaction between the parts of a given system" (Auer, 1994, p. 180).

(13) Citado por Hickerson (1990, p. 25-26).

(14) Sobre esta cuestión la autora pone como modelo el ejemplo canadiense: "Le groupe de travail canadien su les normes a adopté une façon de procéder analogue dans son rapport. Dans un premier temps, il a realisé une enquête dans le milieu afin d'évaluer les méthodes de travail et de connaître les coutumes des archivistes canadiens relativement aux éléments de description des archives. Il fait en même temps l'inventaire des normes existants en archivistique et dans les domaines connexes. Les normes elles-mêmes seront ensuite sanctionnées par la communauté des archivistes et feront l'object de guides d'interprétation et de recueils d'exemples" (Gagnon, 1992, p. 40).

(15) Louise Gagnon-Arguin comparte esta idea, al afirmar que la necesidad de normalizar se ha visto incrementada con la llegada de la informática, para esta autora: "le processus de traitement mécanique d'informations exige la présence de données uniformes et les télécommunications ont accentué ce besoin" (Gagnon, 1992, p. 37).

(16) El incremento del número de documentos en soporte electrónico, y su consideración como documento de archivo, ha influido fuertemente en el desarrollo de pautas específicas para su descripción, específicas para los problemas particulares que plantea el tratamiento de estos materiales (Bailey, 1992 ; Cunningham, 1997).

(17) Un útil resumen de la historia y el contenido de las normas de descripción existentes antes de la publicación de las primeras normas del CIA puede encontrarse en Travesi (1999, p. 11-30). 
(18) Existe una traducción española de esta segunda edición realizada por Carlos Travesí de Diego y difundida a través de la lista de distribución Arxiforum durante el mes de abril de 2000.

(19) Algunos autores norteamericanos han destacado de los tres elementos la necesidad de facilitar el acceso como pieza clave para definir la descripción. Miller afirma que la descripción archivística: "is fundamentally a process of communicating information about sets of records to the potential users" (Miller, 1990, p. 79).

(20) La equiparación de descripción e instrumentos de descripción es una práctica común desde el manual de los archiveros holandeses (Manual..., 1960). También los franceses han equiparado ambos elementos (Baudot, 1970, p. 243-294). Asimismo, Lodolini (1987) se suma a esta corriente. En España, Carmen Pescador (1986, p. 14217) no habla de la descripción, sino directamente de los instrumentos de información; también Michael Cook (1977, p. 114-134), en Gran Bretaña, identificaba la descripción con los instrumentos de descripción.

(21) En este sentido afirma J. Pyeins que una de las ventajas de la aplicación de la informática a la descripción es la flexibilidad de los programas de automatización: "una guía, un inventario, un repertorio, de hecho cualquier instrumento descriptivo, puede concebirse bajo la forma de una base de datos informatizada y eso debería hacerse desde el estadio de su concepción. En efecto, realizado el análisis archivístico del problema nada impide al responsable de la aplicación añadir a la estructura de la grabación lógica de base cualquier elemento que le parezca pertinente para mejorar la precisión de la descripción" (Pyeins, 1991, p. 126).

(22) Realmente, lo que se produce es una revalorización de la información contextual, la información sobre los productores, que adquirirá su propio valor en la norma con la creación de un área específica, el área de contexto (Lodolini, 1987).

\section{Bibliografía}

Auer, Leopold (1994). Standardizing archival terminology. // Archivum. 39 (1994).

Bailey, Catherine (1992). Electronic records and archival description. //Archivaria. 34 (1992) 284-287.

Baudot, M. (1970). Les instruments de recherche. // Association des Archivistes Français. Manuel d'Archivistique : théorie et pratique des archives publiques en France. Paris : SEVPEN, 1970. P. 243-294.

Bearman, David (1989). Description standards : a framework for action. The American archivist. $52: 4$ (1989) 5-17.

Bolt, Nancy (1989). Standards for public libraries. // The library trustee. 4th ed. Chicago : American Library Association, 1988. p. 105

Consejo Internacional de Archivos (1995). ISAD (G) : Norma Internacional General de Descripción Archivística. Adoptada por la Comisión Ad Hoc de Normas de Descripción. Estocolmo, Suecia, 21-23 de enero de 1993 (versión final aprobada por el CIA). Madrid : Dirección General del Libro, Archivos y Bibliotecas, 1995.

Consejo Internacional de Archivos. Comité de Normas de Descripción (2000). ISAD(G). General International Standard Archival Description. Adopted by the Committee on 
Descriptive Standards. Stockholm, Sweden, 19-22 september 1999. 2nd. Ed. Otawa : Consejo Internacional de Archivos, 2000.

Cook, Michael (1977). Archives administration : a manual for intermediate and smaller organizations and for local government. London : Dawson, 1977. P. 114-134.

Cook, Michael ; Procter, Margaret (1989). A Manual of Archival Description. - 2nd. Ed. Aldershot: Gower, 1989.

Cox, Richard J. (1994). Standardizing archival practices : a tool for the information age. // International Congress of Archives (12 ${ }^{\circ}$. 1992. Montreal). Proceedings. // Archivum. 39 (1994).

Crawford, Walt (1986). Technical standards : an introduction for librarians. New York : Knowledge Industry Publications, 1986.

Cunnigham, Adrian (1998). Collecting archives in the next millenium (conferencia presentada en la Conferencia Anual de la Australian Society of Archivists, en julio de 1997 en Adelaida). URL=<http://www.nla.gov.au/nla/staffpaper/acunning7.html> Consultado en septiembre de 1998.

Champagne, Michel ; Chouinard, Denys (1987). Le traitement d'un fonds d'archives : ses documents historiques. Québec : Documentor, 1987.

Checklist of standards for archival description (1989). // The American archivist. $52: 4$ (1989) 479-481.

Dollar, Charles M. (1993). Archivists and records managers in the information age. // Archivaria, 36 (1993) 39.

Dollar, Charles M. (1994). Seizing the opportunity : archivists in the information age. // Archivum. 39 (1994).

Emoine, Jacques (1994). Co-ordination of United Nations Information Systems. // International Congress on Archives (12 ${ }^{\circ}$ 1992. Montreal). Proceedings. //Archivum. 39 (1994) 405-420.

Fishbein, Meyer H. (1981). Automation in archives : a summary history. // ADPA. 3 (1981) 9-13.

Gagnon Arguin, Louise (1992). La normalisation en archivistique : état de la question et problématique. // Couture, Carol (dir.). La normalisation en archivistique : un pas plus dans l'évolution d'une discipline. Quebec : Documentor ; Association des archivistes de Québec, 1992. P. 37

García Gutiérrez, A. (1985). Normalización general y documental : concepto, historia e instituciones. Bol Doc Cienc Inf . 9 (1985) 55-96.

García Martínez, Ana Teresa ; Jiménez Vela, Rosario ; Martín Vega, Consuelo de las Mercedes (1998). La descripción archivística codificada : un formato internacional para la descripción archivística. // Jornadas bibliotecarias de Andalucía (10ª 1998. Jerez de la Frontera) ¿Biblioteca real frente a biblioteca virtual?. Málaga : Asociación Andaluza de Bibliotecarios, D.L. 1998. 411-430.

González de Guzmán, Antonio (1952). Normalización. Madrid : Instituto Nacional de Racionalización del Trabajo, 1952.

González, Pedro (1992). La normalización en la descripción de archivos : un movimiento internacional. // Jornadas sobre metodología para la identificación y valoración de

Scire. 6: 1 (en.-jun. 2000) 55-75. 
fondos documentales de las administraciones públicas (1ª 1991. Madrid). Actas. Madrid : Dirección de Archivos Estatales, 1992.

Guercio, Maria (1994). Models for fostering the development of standard practices within a region or a particular country. // International Congress on Archives (12 ${ }^{\circ} 1992$. Montreal). Proceedings. // Archivum. 39 (1994).

Haworth, Kent M. (194). Standardizing archival description. // International Congress on Archives (12. 1992. Montreal). Proceedings. //Archivum. 39 (1994).

Hensen, Steven (1989). Archives, personal papers and manuscripts : a cataloging manual for archival repositories, historical societies and manuscripts libraries. 2nd. Ed. Chicago : Society of American Archivists, 1989.

Hickerson, H. (1990). Thomas. Standard for Archival Information Management Systems. // The American archivist. 53 : 1 (1990).

Lodolini, Elio (1987). Archivistica : principi e problemi. $4^{\text {a }}$ ed. Milano : Franco Angeli, 1987.

Manual de arranjo e descriçao de arquivos (1960)/ Traduçao de Manoel Adolpho Wanderley. Rio de Janeiro : Arquivo Nacional, 1960.

Manual de Descripción Multinivel (2000). Valladolid : Junta de Castilla y León, 2000. En prensa.

Miller, Fredric M. (1990). Arranging and describing archives and manuscripts. Chicago : Society of American Archivists, 1990. P. 79.

Muñoz Feliu, Miguel C. (195). ISAD $(G)$ : hacia un estándar internacional de descripción archivística. // Métodos de información. 2 : 8 (Noviembre, 1995).

Pescador del Hoyo, Carmen (1986). El archivo : instrumentos de trabajo. Madrid : Norma, 1986, p. 14-217

Favier, Jean (dir.) (1993). La pratique archivistique française. París : Archives Nationales, 1993, p. 141.

Pyeins, J. (1991). Mekanizazioaren ekarpena artxibo ondareen describapenarengan = Contribución de la mecanización en la descripción de fondos de archivo. // Irargi. 4 (1991) 126.

Reglas de catalogación angloamericanas (1983). $2^{\mathrm{a}}$ ed. Washington D.C. : Organización de los Estados Americanos ; San José (Costa Rica) : Universidad de Costa Rica, Biblioteca, Documentación e Información, 1983.

Règles pour la description des documents d'archives (1990). Ottawa : Bureau Canadien des Archivistes, 1990.

Sahli, Nancy (1985). Marc for archives and manuscripts : the AMC format. 2nd. Ed. Chicago : Society of American Archivists, 1985.

Steinmark, Charlotte (1996). The use of information technology in the european searchrooms : results from the danish pilot project Ouverture. //Journal of the Society of Archivists. $17: 1$ (1996).

Szary, Richard V. (1990). Archival applicability of external guidelines for data structures. The American archivist. $53: 1$ (1990) 52-54. 
Szary, Richard (1989). Archival description standards : scope and criteria. // The American archivist. $52: 4$ (1989).

Tarraubella i Mirabet, Xavier (1998). Els arxius i els seus usuaris. // Lligall. 12 (1998) 190-204.

Travesí de Diego, Carlos (1999). La normalización antes de la norma : los Estados Unidos, Canadá y Gran Bretaña. Tabula. 4 (1999) 11-30

Vitali, Stefano (1984). Il dibattito internazionale sulla normalizzazione della descrizione : aspetti teorici e prospettive in Italia. Archivi \& Computer. 4 (1994) 303.

Weber, Lisa B. (1989). Archival description standards : concepts, principles and methodologies. // American Archivist . 52 : 4 (1989).

Working Group on Standards for Archival Description (1989). Report of the Working Group on Standards for Archival Description. // The American archivist. 52: 4 (1989) 456-461. 